\title{
INTELLIGENT VEHICLE SAFETY SYSTEM FOR FACIAL BIOMETRY AND VOICE TO REDUCE TRANSIT ACCIDENTS
}

\author{
Fabio Pires ${ }^{1}$, Eduardo Mario Dias ${ }^{1}$, Miguel Edgar Morales Udaeta ${ }^{1}$ e Felippe da Silva Pires ${ }^{2}$ \\ ${ }^{1}$ Escola Politécnica da USP (POLI-USP) \\ ${ }^{2}$ Escola Superior de Administração e Gestão Strong (ESAGS) \\ E-mails: fabiopires@usp.br, emdias@pea.usp.br, udaeta@pea.usp.br, \\ felippepires1000@gmail.com
}

\section{RESUMO}

$\mathrm{O}$ artigo apresenta uma proposta sobre segurança veicular, pelo uso de sistemas inteligentes de reconhecimento facial e voz de motoristas, que apresentam embriaguez ou cansaços excessivos no volante, como ficar piscando os olhos, abrir e fechar a boca continuamente por bocejos, várias vezes, ou cair a cabeça para baixo ou para cima, assim como de um lado ao outro, demonstrando incapacidade consciente de dirigir um veículo, ou até mesmo de expressões faciais anormais para um motorista consciente em trânsito. O sistema é composto por uma câmera interna para verificação e reconhecimento de expressão facial, com um microfone embutido para reconhecimento de voz normal, sem intermitência na voz, demonstrando embriaguez ou sonolência. O sistema é configurado com algoritmos que permitem verificar a simetria facial em quadrantes, eixos horizontais e verticais, além de expressões faciais codificadas e frequência de voz. Qualquer anormalidade faz o sistema entrar em operação, não permitindo ligar o carro, ou caso esteja em movimento, dispara alarmes sonoros e reduz a velocidade do veículo até parar. Com o sistema implantado haverá diminuição significativa de mortes e acidentes de trânsitos, visto que no Brasil, segundo a Organização das Nações Unidas, ultrapassam mais de 47 mil mortes anuais por acidentes veiculares, além de custos significativos para o sistema de saúde público e privado.

Palavras-chave: sistema inteligente veicular, segurança veicular integrada, biometria facial, redução de acidentes no trânsito, tecnologia automotiva.

\begin{abstract}
The article presents a proposal on vehicular safety, the use of intelligent facial recognition systems and drivers' voice, which show drunkenness or excessive fatigue in the steering wheel, such as blinking the eyes, opening the mouth several times, yawning, or falling over the head down or up, as well as from one side or the other, demonstrating a conscious inability to drive a vehicle, or even from abnormal facial expressions to a conscious driver in transit. The system consists of an internal camera for facial expression verification and recognition, with a built-in microphone for normal speech recognition, with no voice blinking, demonstrating drunkenness or drowsiness. The system is configured with algorithms that allow verifying the facial symmetry in quadrants, horizontal and vertical axes, as well as coded facial expressions and voice frequency. Any abnormality causes the system to come
\end{abstract}


into operation, not allowing the car to start, or if it is in motion, triggers audible alarms and slows the vehicle to a stop. With the system in place there will be a significant decrease in deaths and traffic accidents, since in Brazil, according to the United Nations, more than 47 thousand deaths per year are caused by vehicular accidents.

Keywords: vehicle intelligent system, integrated vehicular safety, facial biometry, reduction of traffic accidents, automotive technology.

\section{INTRODUÇÃO}

O sistema de segurança veicular, pelo uso de sistemas inteligentes de reconhecimento facial e voz de motoristas tem por objetivo reduzir os acidentes de trânsito por embriaguez ou cansaços excessivos no volante. O controle interno é composto por um sistema inteligente integrado que poderá estar interligado ou não ao computador de bordo, que verifica a imagem da mini câmera pela movimentação da cabeça e expressão facial, com integração mutua de voz codificada e vídeo, como também a frequência e modulação de voz do motorista, com sistema comparativo entre a voz normal ou anormal do motorista, antes de dar a partida na ignição do veículo ou depois. Se houver diferença significativa comparativa entre os parâmetros pré-estabelecidos na voz e nos movimentos anormais da cabeça, como apresentando sinais de sonolência, o sistema dará um alerta sonoro e limitará a velocidade do veículo (PIRES et al, 2014).

A metodologia de pesquisa é exploratória pelas bibliografias existentes e também pelo case sobre tecnologia de biometria facial FACEID da empresa Imagesec.

Os acidentes de trânsitos nas rodovias municipais, estaduais e federais são problemas gravíssimos que atinge todos os países globalmente e algumas ações se fazem necessárias para mitigar estas fatalidades. Estima-se 3.400 mortes por dia e de 20 milhões a 50 milhões de feridos por ano no mundo (ALMEIDA et al, 2004).

São apresentadas as tecnologias de seguranças utilizadas, como a veicular interna, biometria facial, funcionalidade operacional do sistema, controle vertical e horizontal do movimento da face, sistema integrado de VoIP e Vídeo, redução de acidentes de trânsitos e considerações sobre este problema crítico nacional e global.

\section{SISTEMA INTELIGENTE VEICULAR}

O artigo apresenta uma proposta sobre segurança veicular, pelo uso de sistemas inteligentes de reconhecimento facial e voz de motoristas, que apresentam embriaguez ou cansaços excessivos no volante, como ficar piscando os olhos, abrir e fechar a boca continuamente por bocejos, várias vezes, ou cair a cabeça para baixo ou para cima, assim como de um lado ao outro, demonstrando incapacidade consciente de dirigir um veículo, ou até mesmo de expressões faciais anormais para um motorista consciente em trânsito. O sistema é composto por uma câmera interna para verificação e reconhecimento de expressão facial, com um microfone embutido para reconhecimento de voz normal, sem intermitência na voz, demonstrando embriaguez ou sonolência. O sistema é configurado com algoritmos que permitem verificar a simetria facial em quadrantes, eixos horizontais e verticais, além de expressões faciais codificadas e frequência de voz. Qualquer anormalidade faz o sistema entrar em operação, não permitindo ligar o carro, ou caso esteja em movimento, dispara alarmes sonoros e reduz a velocidade do veículo até parar. 


\subsection{Tecnologia Veicular Interna e Biometria Facial}

A tecnologia utilizada no sistema de interação homem-máquina é composta por uma câmera de vídeo de alta resolução, um microfone embutido, um módulo eletrônico de comunicação digital e acessórios existentes no veículo, como alto falantes e multimídia. Tem por objetivo integrar as tecnologias envolvidas e permitir a interpretação das informações advindas para reconhecimento facial e de voz.

A biometria facial é uma técnica que consiste em analisar e realizar o conhecimento da face através da geometria facial e o sistema utilizado será o FACEID, um software de reconhecimento facial da empresa de solução de segurança Imagesec, que opera com câmeras de vídeos IP, no padrão ONVIF. O sistema reconhece até 66 pontos de uma face, desde que a imagem permita a identificação e sejam definidos os pontos, como mostrado na figura 01, e estejam interligados entre si, para cada polígono um código digital é gerado. O software é confiável, gerando alto desempenho de informações (IMAGESEC, 2018).

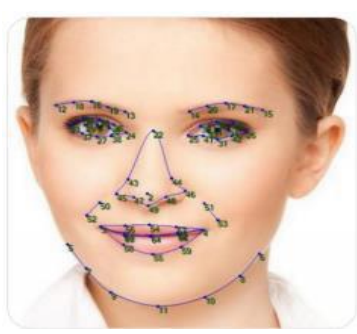

Figura 01. Combinações pontuais para monitoramento de biometria facial. IMAGESEC, 2018.

\subsection{Segurança Veicular Integrada}

Com o sistema de segurança veicular integrada ao sistema eletrônico do carro é possível fazer o controle contínuo de segurança facial pela câmera embutida no teto ou integrado no retrovisor. Com a implantação do sistema haverá diminuição de mortes e acidentes de trânsitos, sendo este um grande problema para o sistema de saúde público e privado no Brasil. $\mathrm{Na}$ figura 02, a câmera está continuamente monitorando as expressões faciais do condutor, como também, o movimento anormal da cabeça para os lados ou para cima ou para baixo.

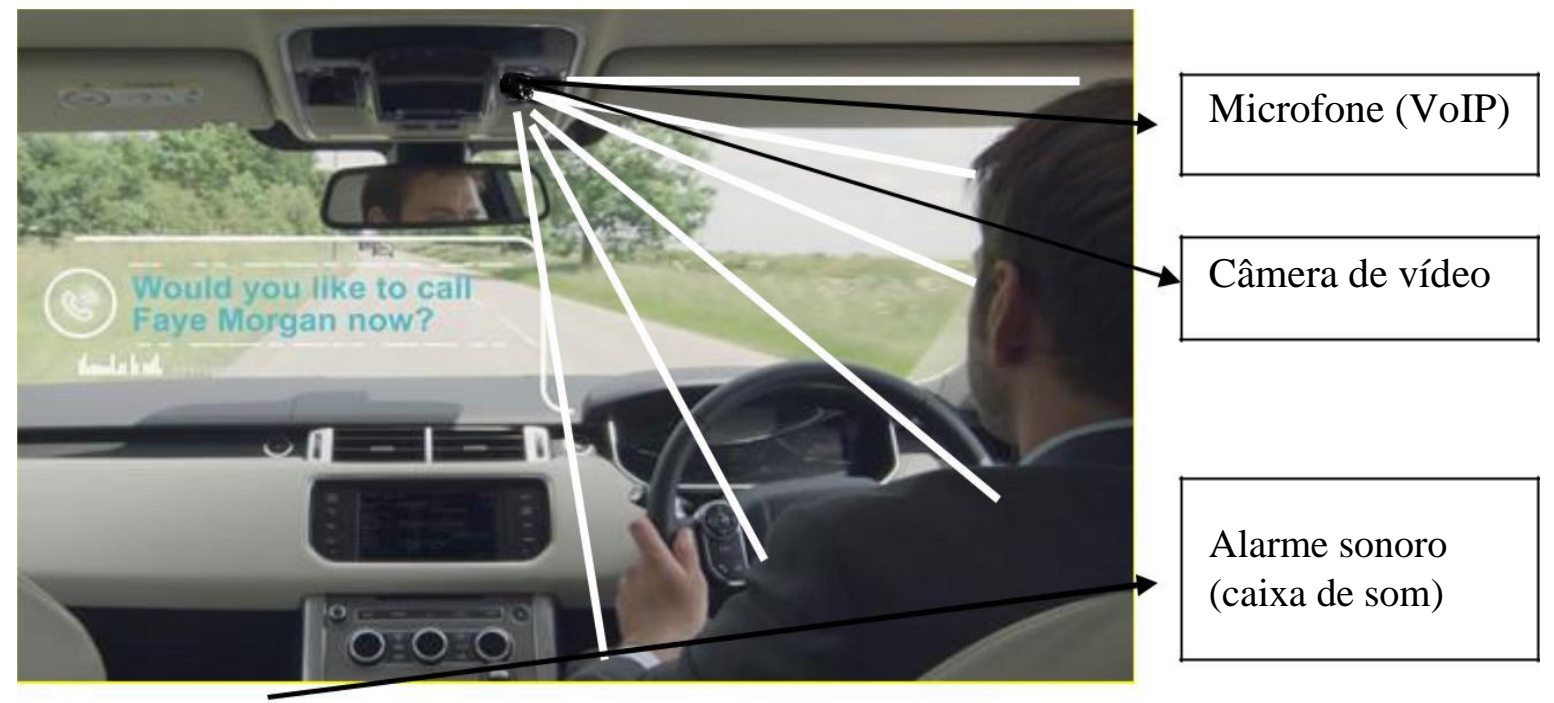

Figura 02. Interação câmera x motorista. Fonte: Divulgação, Jaguar Land Rover (adaptado). 
A posição do motorista, em condições normais, fica na vertical e quando está em condições de anormalidade de atenção no trânsito pode sair do eixo vertical tendendo para direita ou esquerda até um limite aceitável de dirigibilidade.

Muitas empresas objetivando a segurança, inclusive bancos, se utilizam de tecnologia de biometria facial para fazer controle de acesso de pessoas, assim como acesso à informação confidencial, pessoal e sigilosa.

\section{FUNCIONALIDADE OPERACIONAL DO SISTEMA}

Muitas empresas objetivando a segurança, inclusive bancos, se utilizam de tecnologia de biometria facial para fazer controle de acesso de pessoas, à informação confidencial, pessoal e Sigilosa, assim como confirmação de titularidade. Aproveitando-se de tecnologias existentes foram desenvolvidos algoritmos que permitem a interação do sistema FACEID com o módulo de segurança para alarmes sonoros e controle de velocidade.

$\mathrm{Na}$ figura 03 é utilizado a tecnologia de biometria facial para comparar e comprovar se o usuário do cartão é o titular da conta.

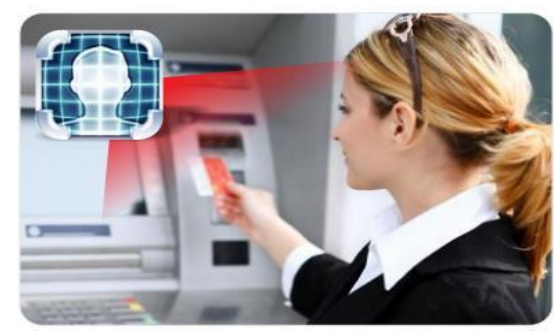

Figura 03. Biometria facial em caixa eletrônico. Fonte: Imagesec, 2018.

Utilizando-se da mesma tecnologia da biometria facial, mas com algoritmos lógicos de integração para o controle integrado de situação de risco (na condução de veículos) são utilizados parâmetros gráficos multidimensionais de posição passiva relativa e dinâmica, conforme figuras 04 (movimento vertical) e 05 (movimento horizontal).

\subsection{Controle vertical do movimento da cabeça}

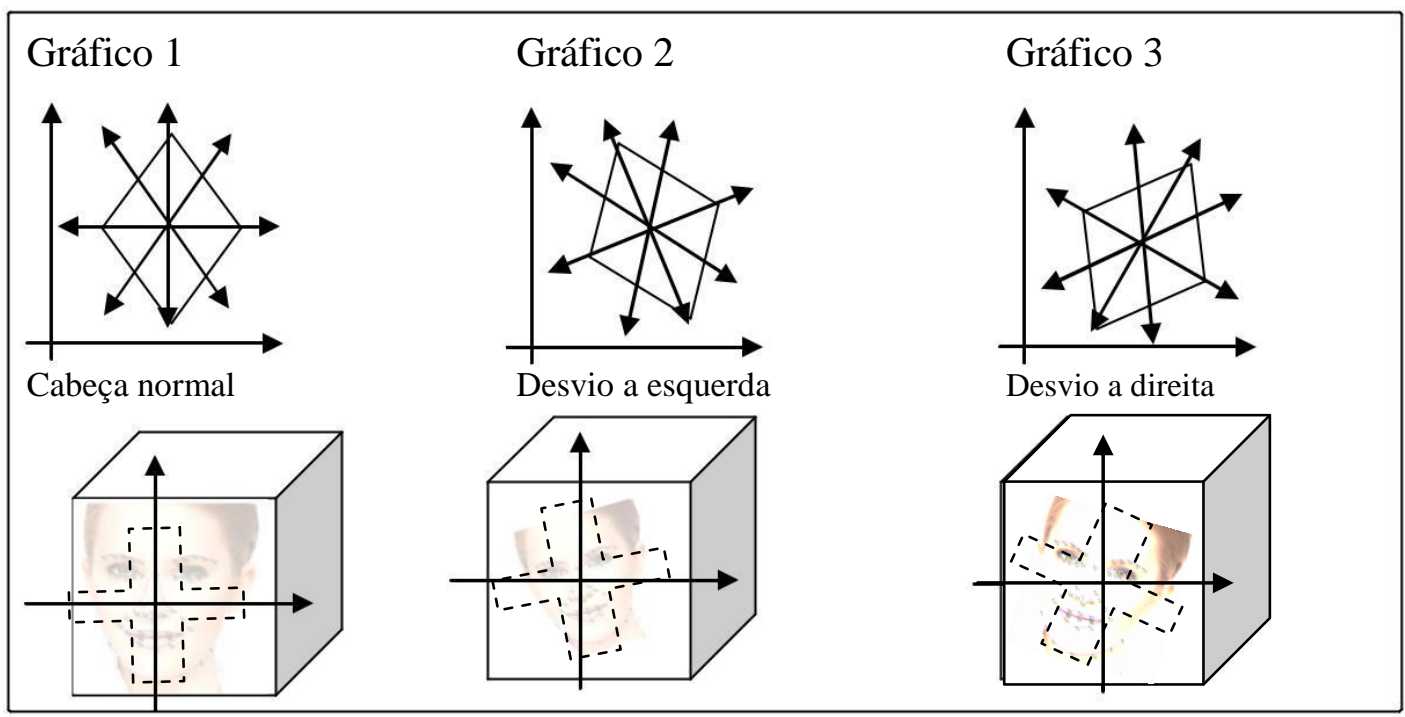

Figura 04. Controle vertical da cabeça. 


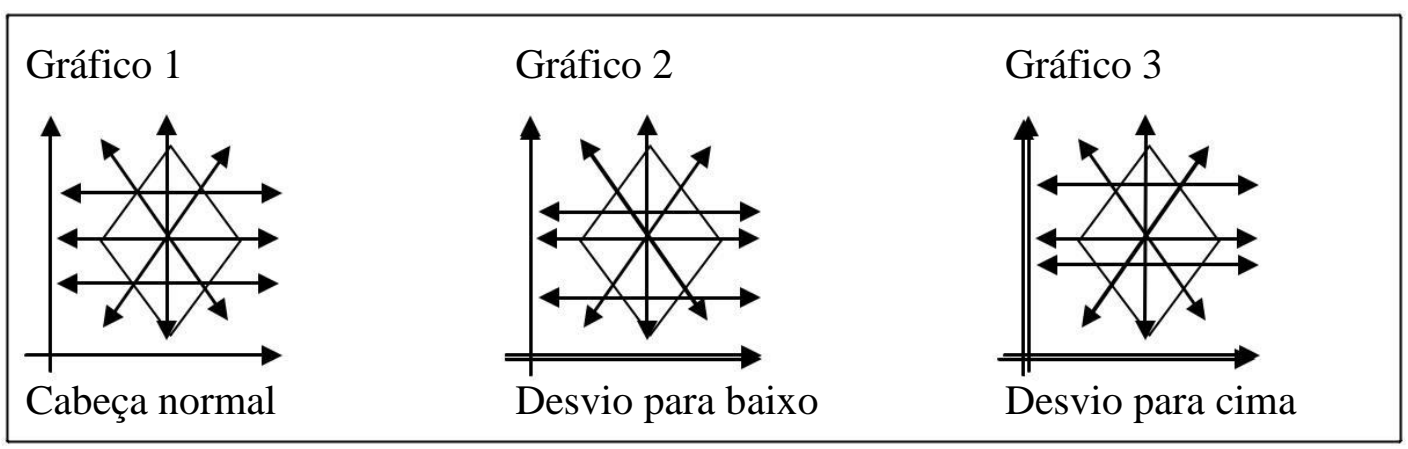

Figura 05. Controle horizontal da cabeça.

O princípio é o mesmo do controle longitudinal da cabeça, em que existem pontos entre a face horizontal facial do rosto, tanto em relação a posição dos olhos, quanto da boca. Qualquer variação significativa poderá disparar alarmes sonoros para despertar ou avisar o motorista. Utilizando-se do mesmo raciocínio o sistema é acionado pelo fechar dos olhos em tempos maiores de 1 segundo.

\section{SISTEMA INTEGRADO DE VOIP E VÍDEO}

O sistema integrado de vídeo e voz recebe as informações da câmera de vídeo interna, a voz do microfone e processa os dados para possíveis respostas em alarme sonoro, buzina e redução de velocidade, se necessário (figura 06).

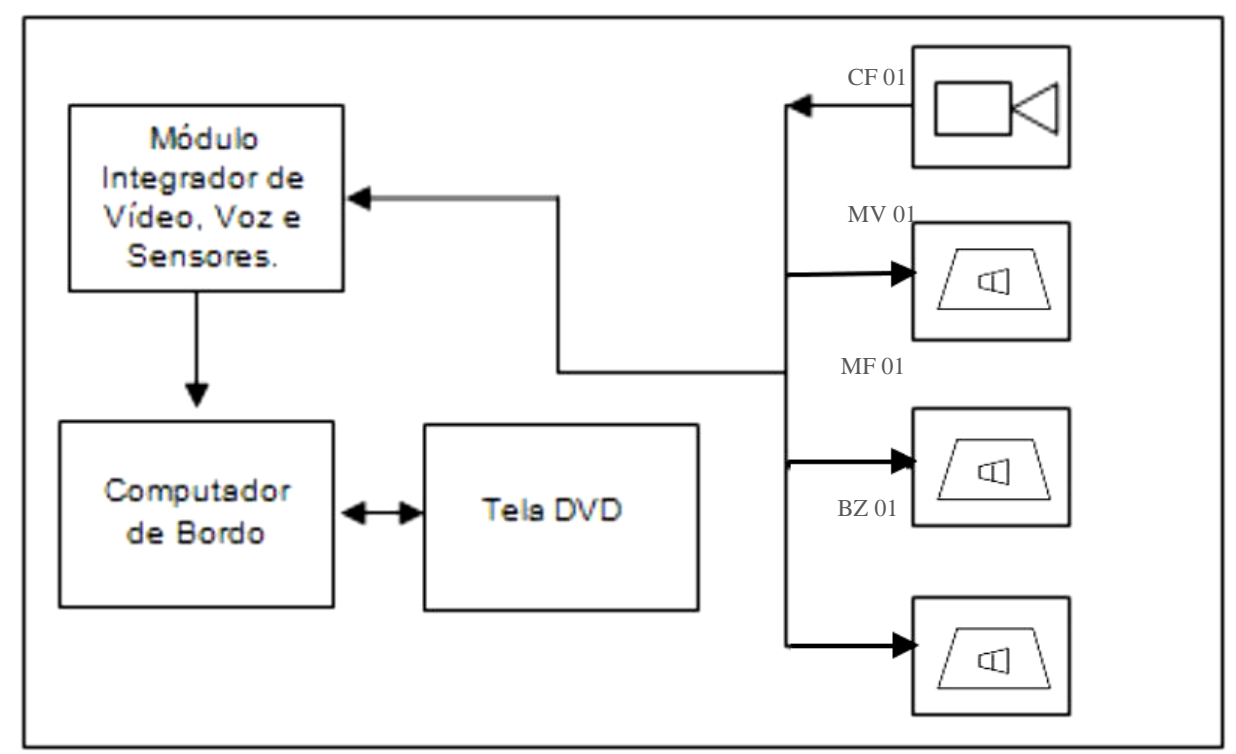

Figura 06. Diagrama do sistema integrado de vídeo e VoIP.

\section{REDUÇÃO DE ACIDENTES NO TRÂNSITO}

Vários fatores contribuem para o acidente de trânsito, dentre os quais os fatores humanos, que é o objeto de pesquisa, cuja interação homem-máquina possibilita redução significativa de acidentes, fatores viários ambientais, que depende do estado das vias de acessos e escoamento de trânsito e o meio ambiente que estão inseridos, fatores veiculares, que dependem de seu estado de manutenção, aerodinâmica e/ou falhas mecânicas ou elétricas e fatores legais e governamentais. 
A figura 07 apresenta uma estimativa dos fatores que contribuem para acidentes de trânsito.

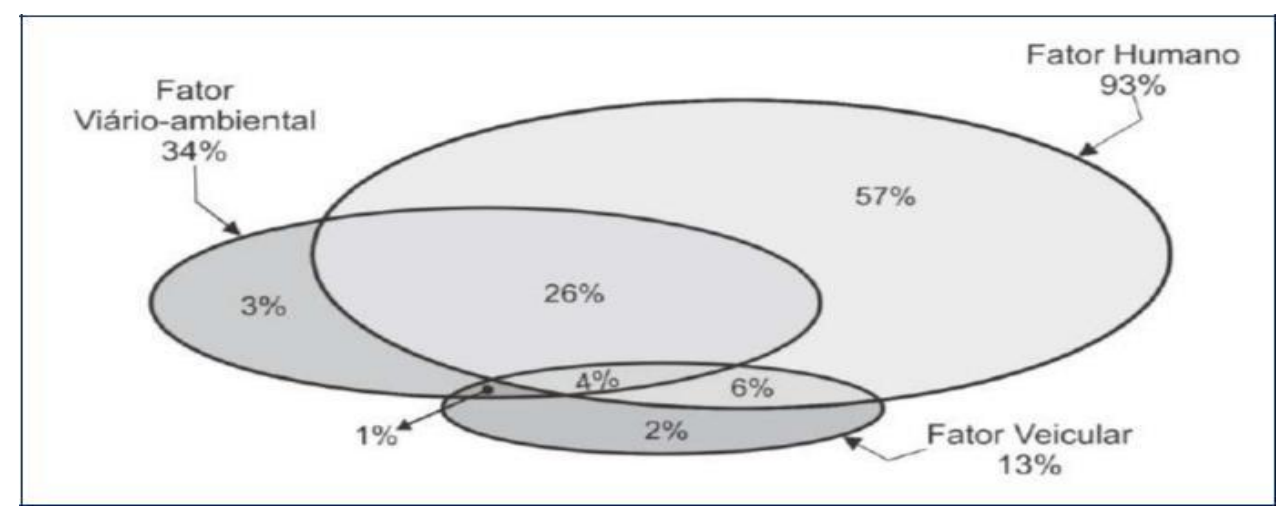

Figura 07 . Fatores que contribuem para o acidente de trânsito.

Taxa de mortalidade em acidentes de trânsito entre adolescentes e jovens de 15 a 19 anos

\begin{tabular}{|c|c|c|c|c|c|c|c|c|c|c|c|}
\hline UF & 2000 & 2001 & 2002 & 2003 & 2004 & 2005 & 2006 & 2007 & 2008 & 2009 & Média \\
\hline Rondônia & 14 & 18,1 & 22,7 & 16,3 & 24,4 & 15,4 & 22,9 & 22 & 25,2 & 25,5 & 20,65 \\
\hline Acre & 3 & 11,6 & 18,3 & 26 & 10,1 & 8,6 & 12,7 & 9,4 & 12,7 & 9,8 & 12,22 \\
\hline Amazonas & 8,1 & 7,6 & 9,9 & 6,6 & 9 & 8,8 & 11 & 6,7 & 12,4 & 7,8 & 8,79 \\
\hline Roraima & 34,6 & 25,4 & 39,1 & 40,1 & 11 & 19 & 16,4 & 18,3 & 23,4 & 18,5 & 24,58 \\
\hline Para & 10,5 & 11,6 & 12,2 & 13,8 & 10,5 & 13 & 11,1 & 14,8 & 13,8 & 12,7 & 12,4 \\
\hline Amapá & 15,7 & 15 & 28,8 & 23,1 & 5,7 & 8,3 & 14,9 & 14,2 & 8,9 & 10,3 & 14,49 \\
\hline Tocantins & 20,8 & 17,4 & 19,1 & 22,9 & 22,6 & 18,8 & 23,5 & 20,4 & 20,6 & 30 & 21,61 \\
\hline Maranhão & 6,5 & 7,6 & 10,2 & 8,3 & 9,5 & 9,8 & 11,8 & 14,8 & 21,2 & 15,7 & 11,54 \\
\hline Piauí & 10,1 & 11,7 & 14,7 & 13,9 & 12,8 & 13,1 & 28,5 & 16,5 & 19,3 & 23,7 & 16,43 \\
\hline Ceara & 13,4 & 14,8 & 14 & 15,7 & 15,2 & 16,3 & 17,5 & 17,1 & 17,3 & 15,3 & 15,66 \\
\hline Rio Grande do Norte & 15,6 & 11,8 & 13,2 & 13 & 8,4 & 10 & 17,4 & 12,4 & 13,4 & 11,7 & 12,69 \\
\hline Paraíba & 10 & 8,9 & 17 & 10,6 & 17,6 & 16,5 & 24,3 & 18,3 & 24,7 & 21,8 & 16,97 \\
\hline Pernambuco & 16,4 & 12,4 & 13,6 & 11,4 & 14,4 & 12,7 & 17,6 & 14,2 & 14,4 & 15,5 & 14,26 \\
\hline Alagoas & 15,2 & 12,6 & 13 & 13,5 & 14 & 13 & 14,1 & 18 & 17,5 & 14,8 & 14,57 \\
\hline Sergipe & 13,2 & 17,2 & 19,3 & 13,9 & 20,6 & 11,9 & 18,5 & 16,8 & 19,2 & 15,3 & 16,59 \\
\hline Bahia & 6,9 & 6,4 & 8,1 & 7,2 & 7,3 & 7,8 & 9,6 & 10 & 10,1 & 11 & 8,44 \\
\hline Minas Gerais & 10,6 & 12,6 & 14 & 13 & 13,8 & 13,5 & 18,3 & 17,8 & 19,6 & 15,7 & 14,89 \\
\hline Espirito Santo & 21,4 & 18,6 & 26 & 18,2 & 18,9 & 17,1 & 18,8 & 25,2 & 28,2 & 23 & 21,54 \\
\hline Rio de Janeiro & 14,1 & 13,3 & 16,7 & 17,9 & 14,4 & 13,7 & 19,4 & 16,6 & 15,7 & 12,9 & 15,47 \\
\hline São Paulo & 13,9 & 17,4 & 15,6 & 15,4 & 15,4 & 16 & 18,9 & 20,4 & 21,7 & 17,2 & 17,19 \\
\hline Paraná & 25,1 & 22,8 & 24,4 & 27,6 & 28 & 27 & 29,5 & 30,5 & 33,4 & 28,4 & 27,67 \\
\hline Santa Catarina & 31,2 & 29,5 & 32,1 & 31,6 & 37,9 & 36,9 & 37,6 & 41,9 & 34,3 & 31,2 & 34,42 \\
\hline Rio Grande do Sul & 18,4 & 15,5 & 18,4 & 16,4 & 17,1 & 16,8 & 16,8 & 18,1 & 18,3 & 21,5 & 17,73 \\
\hline Mato Grosso do Sul & 17,9 & 20,3 & 26,2 & 18,4 & 21,3 & 22,6 & 25,3 & 26,9 & 28,1 & 24,1 & 23,11 \\
\hline Mato Grosso & 27,5 & 22,4 & 35,5 & 23,4 & 27,4 & 27,5 & 24,7 & 26,6 & 26,6 & 31,2 & 27,28 \\
\hline Goiás & 26,3 & 21,9 & 20,1 & 19,3 & 25,1 & 21,9 & 23,4 & 28,9 & 29,1 & 25,2 & 24,12 \\
\hline Distrito Federal & 23,1 & 18,2 & 16,5 & 13,3 & 15,9 & 12,4 & 11,5 & 14,9 & 6,9 & 17,5 & 15,02 \\
\hline
\end{tabular}

Fonte: IBGE, 2018. 
A Organização Mundial da Saúde (OMS) lançou em maio de 2011 a Década de Ação pela Segurança no Trânsito (2011-2020), em 178 países, sobre mortes por acidentes de trânsito, em que os governos de todos os continentes se comprometem a tomar novas medidas concretas para prevenir os acidentes no trânsito, que matam mais de 1,25 milhão de pessoas por ano no mundo (ONU, 2018).

No Brasil, o Projeto está sendo desenvolvido em várias cidades, com a participação da OPAS/OMS, e aporte técnico e financeiro do Governo Federal.

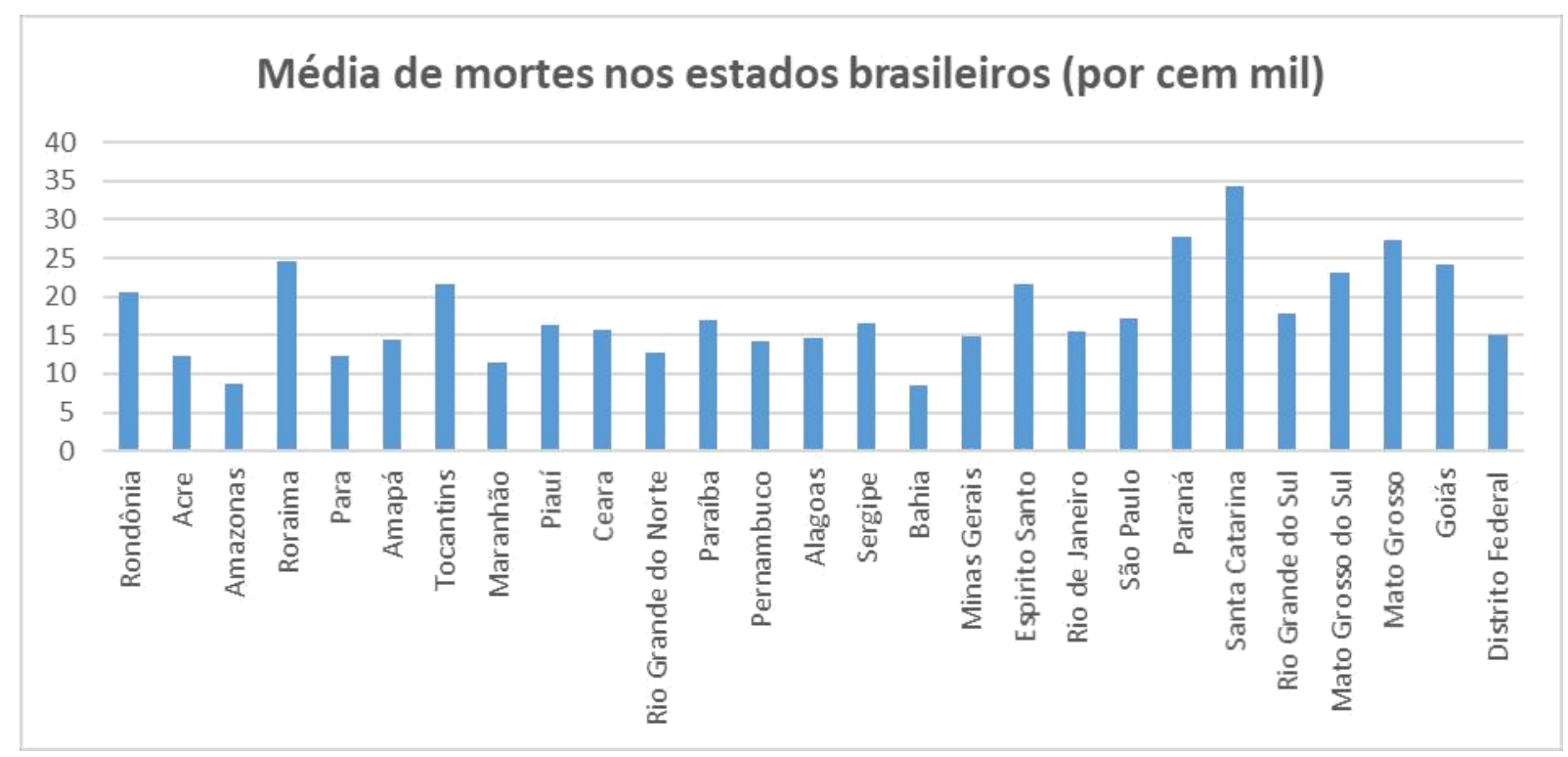

Fonte: IBGE, 2018.

\section{CONCLUSÃO}

Existe um problema seríssimo no país, em relação aos acidentes de trânsitos, sendo em grande parte ocasionados por descuidos, imprudências e negligências dos condutores, principalmente por aqueles que dormem na direção ou estão embriagados e/ou drogados. E para resolver parte deste problema foi apresentado um sistema que permite verificar as condições normais do motorista, seja pela expressão facial, frequência de voz ou comportamento de sonolência e apresentando qualquer anormalidade faz o sistema entrar em operação, não permitindo ligar o carro, em caso de embriagues, ou caso o veículo esteja em movimento, dispara alarmes sonoros e reduz a velocidade do veículo até parar. Com o sistema implantado haverá diminuição significativa de mortes e acidentes de trânsitos, diminuindo os custos para o sistema de saúde brasileiro tanto privado quanto público.

\section{REFERÊNCIAS}

PIRES, F.; DIAS, E.M ; FONTANA, C. F. ; PIRES, F. S. . Integrated intelligence system in urban automobile communication. In: Simpósio Internacional Automotivo SIMEA 2014, 2014, São Paulo. INOVAR ? AUTO E A EVOLUÇÃO TECNOLÓGICA DO BRASIL, 2014. 
ALMEIDA, Lívia Victório de Carvalho, PIGNATTI, Marta Gislene, ESPINOSA; Mariano Martinez. Main factors associated with motor vehicle accidents on Federal Highway 163. Disponível em: <http://www.scielo.br/pdf/csp/v25n2/08.pdf>. Acesso em 10/03/2018.

BRASIL, Senado Federal. Estudo da organização mundial da saúde OMS sobre mortes por acidentes de transito em 178 países e base para década de ações para segurança. Disponível em: <http://www.senado.gov.br/noticias/Jornal/emdiscussao/motos/ saude/estudo-da-organizacao-mundial-da-saude-oms-sobre-mortes-por-acidentes-de-transitoem-178-paises-e-base-para-decada-de-acoes-para-seguranca.aspx>. Acesso em 12/03/2018.

BRASIL, Senado Federal. Acidentes de trânsito no Brasil. Disponível em: <http://www.senado.gov.br/noticias/Jornal/emdiscussao/upload/201204\%20-\%20novembro/ ed13_imgs/ed13_p21_info.jpg>. Acesso em 18/04/2018.

ONU. Manual para fortalecer a legislação de segurança no transito Disponível em: $<$ https://nacoesunidas.org/oms-lanca-manual-para-fortalecer-a-legislacao-de-seguranca-notransito/>. Acesso em 22/04/2018.

BRASIL, IBGE. Dados estatísticos sobre mortes entre jovens de 15 a 19 anos. Disponível em <https://seriesestatisticas.ibge.gov.br/lista_tema.aspx?op=2\&no=4>. Acesso em 08/04/2018. 